This article has been scanned by iThenticat No plagiarism detected

Volume 3, Issue 4, August 2021

p. $278-285$

\title{
THE REALITY OF E-LEARNING PERFORMANCE IN THE MINISTRY OF HIGHER EDUCATION AND ITS RELATIONSHIP TO INDICATORS SUSTAINABLE KNOWLEDGE (AL BURAIMI UNIVERSITY COLLEGE AS A MODEL)
}

http://dx.doi.org/10.47832/2757-5403.4-3.24

\section{Fatma Hamed Mohammed ALNAIMI'1 \& Falah Mohammed ALJABRI²}

\begin{abstract}
:
The study aimed at the reality of education in the Ministry of Higher Education (Al-Buraimi University College as a model) and its relationship to indicators of sustainable knowledge, and to achieve the objectives of the study the descriptive analytical approach, a set of study objectives from all Buraimi College students for the academic year (2021/2020) and their adults according to: Personnel Affairs Department ( 3500) male and female students, while the study sample consisted of (347) male and female students distributed among the faculties of the University College.

The researcher used the questionnaire tool, which consisted of two areas: The first section: E-learning, and it consisted of (4) domains: the effectiveness of the content of education, the effectiveness of teaching through e-learning, the effectiveness of education through e-learning, the effectiveness of e-learning, e-learning, while dealing with The second section: digital knowledge in the field of knowledge (4) are the areas: knowledge, media and media, outputs and objectives, and to obtain the results the researchers used the SPSS program and the following scientific methods: percentages and frequencies, Cronbach's alpha test, correlation coefficient. In light of the results of the study, the researchers recommended the following: Providing more internet speed for college students and professors for free, in addition to using the freely available electronic teaching aids.
\end{abstract}

Key words: E-Learning, Sustainable Knowledge, Indicators.

\footnotetext{
${ }^{1}$ Dr. , Islamic Science University, Malaysia, Fatma.alnaaimi@gmail.com, https://orcid.org/0000-0002-8719$\underline{7285}$

${ }^{2}$ Researcher, Islamic Science University, Malaysia, falah@imco.edu.om, https://orcid.org/0000-0002-8132$\underline{5450}$
}

Copyright (C) Published by IJHER Journal, www.ijherjournal.com Rimar Academy, Fatih, Istanbul, 34093 Turkey

All rights reserved 


\title{
واقع أداء التعليم الإكتروني في وزارة التعليم العالي وعلاقته بمؤشرات المعرفة المستدامةً (كلية البريمي الجامعيةً أنموذجاً)
}

\author{
3 \\ فاطمة حمد محمد النعيمي
}

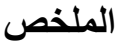

هدفت الدراسة إلى التعرف على واقع أداء التعليم الإلكتروني في وزارة التعليم العالي (كلية

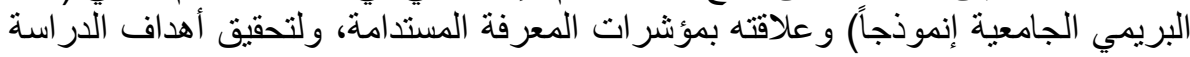

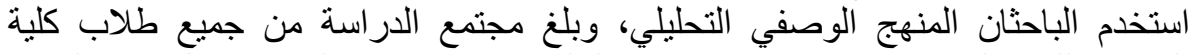

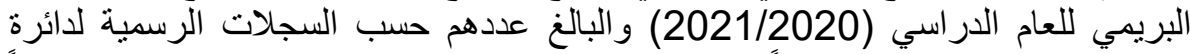

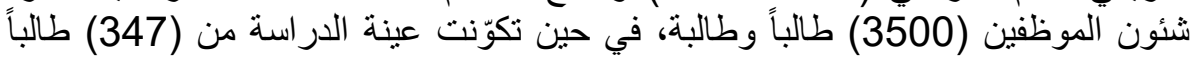
وطالبة موز عين على كليات الكلية الجامعية.

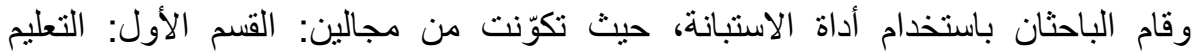

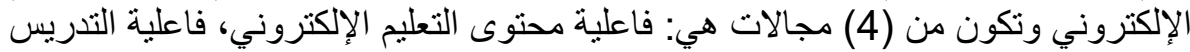

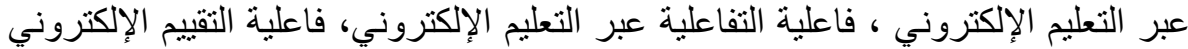

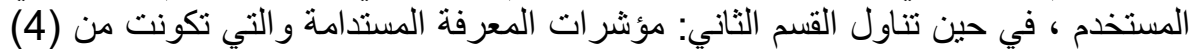

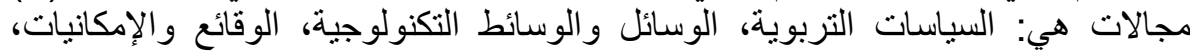

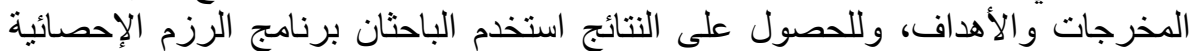

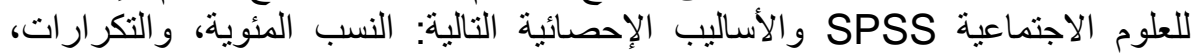

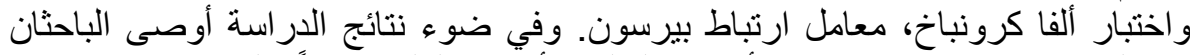

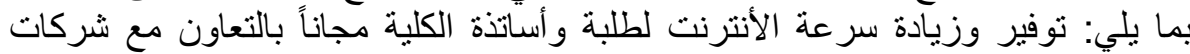

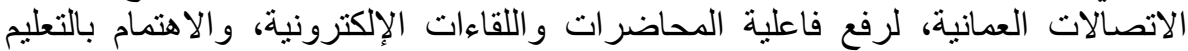

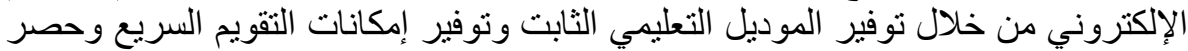

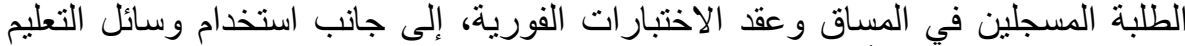

الإلكترونية المتاحة مجاناً. الكلمات المفتاحية: التعليم الإكتروني، المعرفة المستدامة، المؤشرات.
\end{abstract}

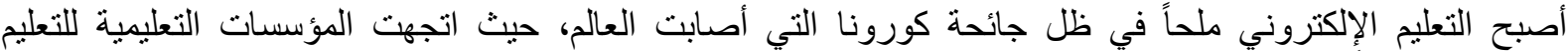

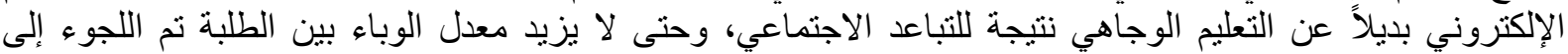

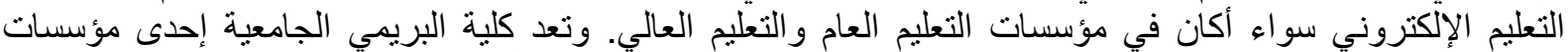

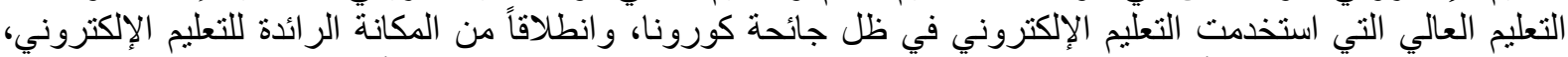

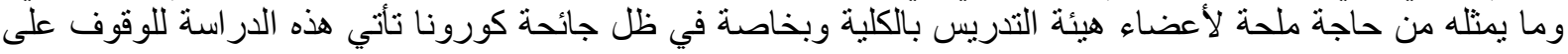

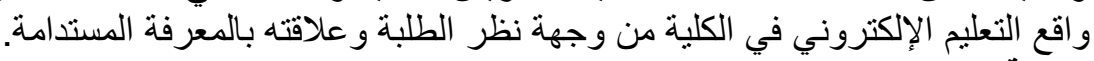

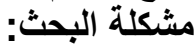
تبحث الدراسة في سبل نشر المعرفة وإعادة بناءها من أجل تحسين خصائص التعليم والاستفادة من وسائل اكتساب

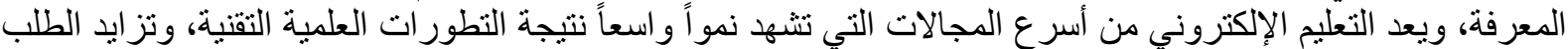

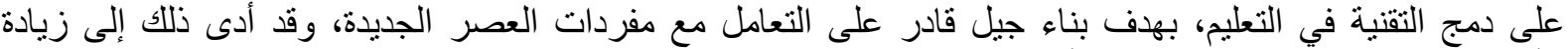

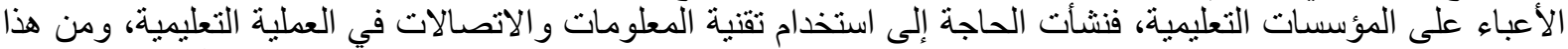

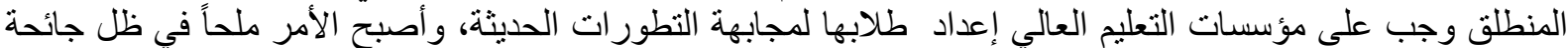

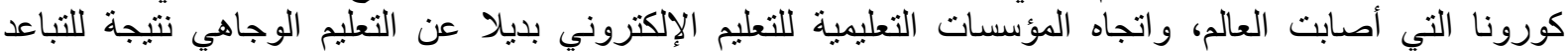

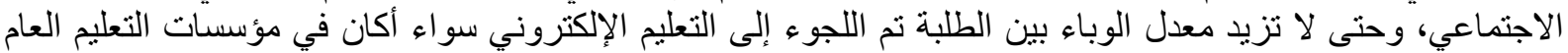

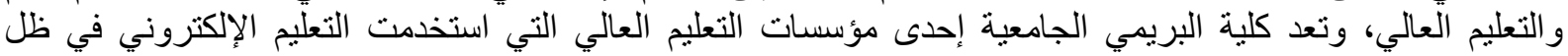


THE REALITY OF E-LEARNING PERFORMANCE IN THE MINISTRY OF HIGHER

EDUCATION AND ITS RELATIONSHIP TO INDICATORS SUSTAINABLE

KNOWLEDGE (AL BURAIMI UNIVERSITY COLLEGE AS A MODEL)

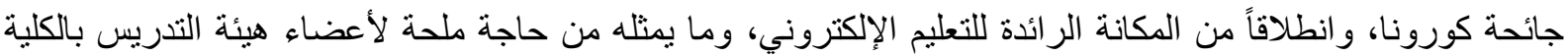

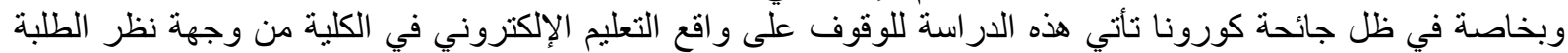
و وعلاقته بالمعرفة المستدامة.

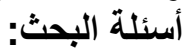

تحاول الدراسة الإجابة عن الأسئلة التالية:

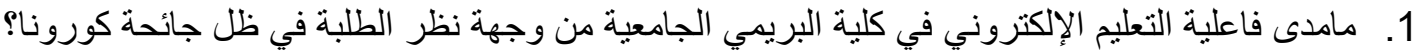

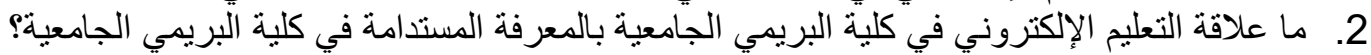

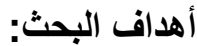

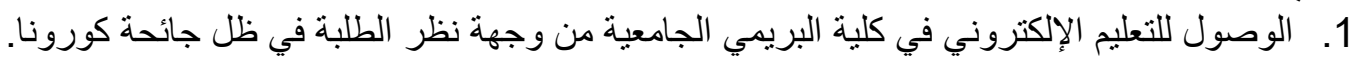
2. تحليل العلاقة بين التعليم الإلكتروني و المعرية اليرية المستدامة في كلية البريمي الجامعية. أهمية البحث:

1. السعي إلى تشخيص واقع التعليم الإلكتروني في كلية البريمي الجامعية بالاعتماد على آراء الطلبة، إذ يعد التعليم

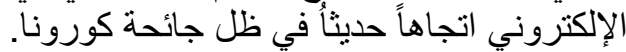

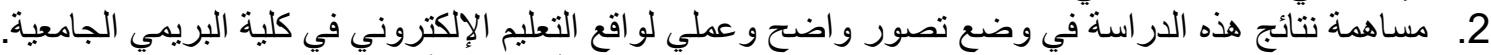

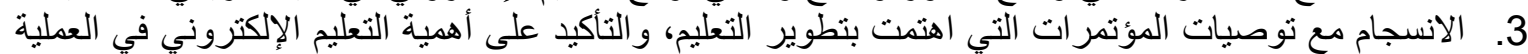
التعليمية بهدف الوصول تلى إلى مستويات ومر احل أفضل تضمن استمر ارية المعرفة وديمو التهنها بشكل منميز. منهج البحث: أنبع البحث المنهج الوصفي التحليلي الإحصائي. مصادر البيانات: نتمثل في: البعة المصادر الأولية: الاستبانة، المصادر الثنانوية: الكتب_المر اجع-التقارير -المجلات. الحدود الموضوعية للبحث: الحدود الموضو عية: اقتصرت البحثة الدر اسة واقع التعليم الإلكتروني في كلية البريمي الجامعية التقنية.

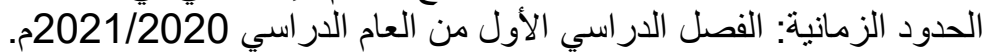

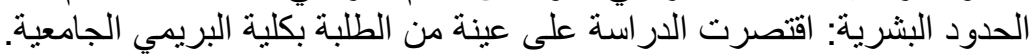

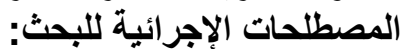
التعليم الإلكتروني: هو التبعليم الذي بيتخدم فيه عضو هيئة التجريس في كلية البريمي الجامعية الوسائط الإلكترونية

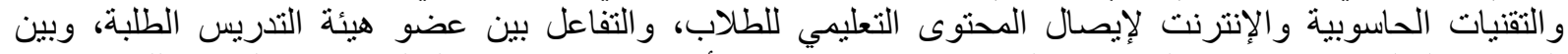

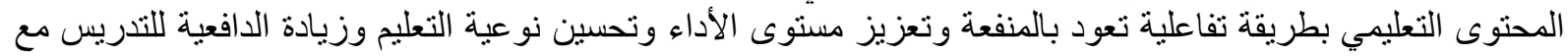
بذل الجهد القليل في الاستخدام. كلية البريمي الجامعية: كلية جامعية خاصة تلفية الإنح الطلبة درجتي البكالوريوس والدبلوم في التخصصات العلوم الإنسانية و التجارية ونظم المعلومات و القانون.

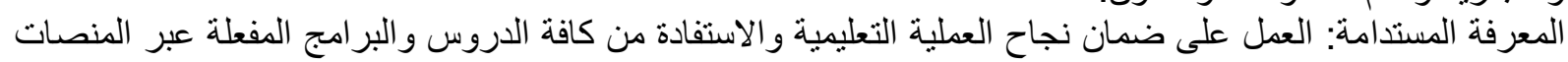

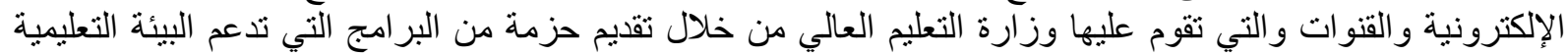

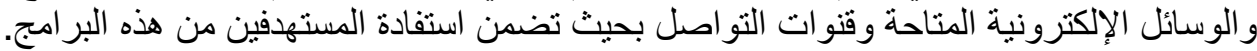
الإطار النظري والإلكرونية الاستات السابقة:

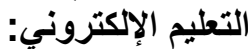
هو التعليم المعتمدي على الحاسوب وشبكة الإنترنت لتوصيل المحتوى التعليمي للمتعلمين عبر التو اصل بين المتعلم والمعلم،

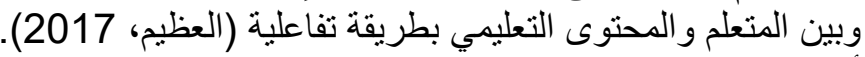

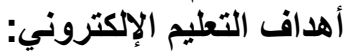

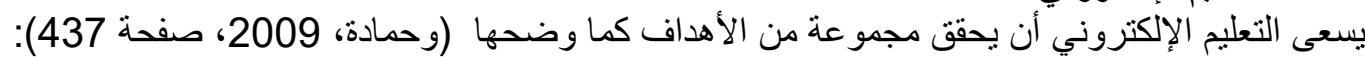

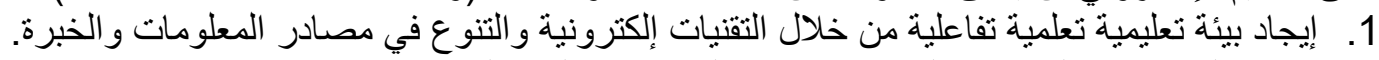
3.

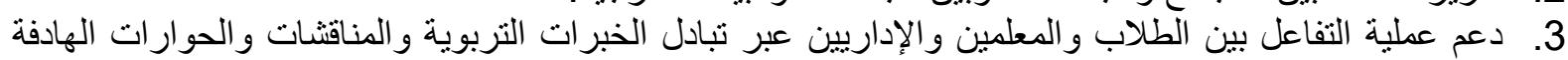

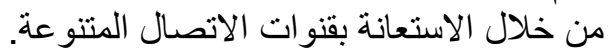

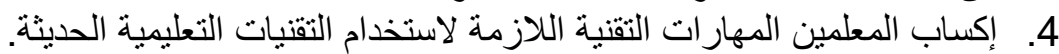

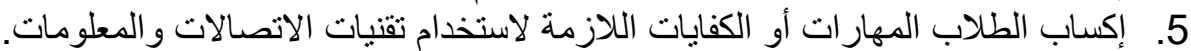
6. تطوير دور المدرس في العملية التعليمية بحيث نواكبة لاكب التطور اتلنيات العلمية والتكنولوجية المستمرة. 
7. المساهمة في إنشاء بنية تحنية وقاعدة من تقنية المعلومات التي تقوم على الأسس الثقافية بهدف إعداد مجتمع جديد لمتطلبات القرن الحادي و العشرون. 8. تتمية الاتجاهات الإيجابية للمتعلمين و القائمين على عملية التعليم وأولياء الأمور و المجتمع ككل نحو تقنية المعلومات

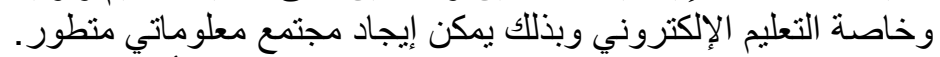

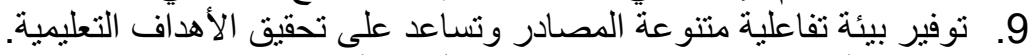

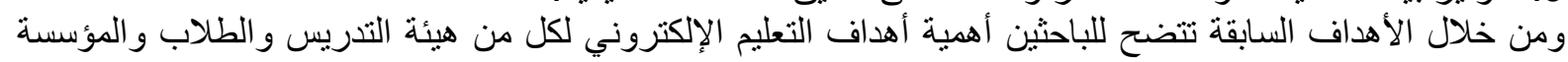
التعليمية و المجتمع. الإن.

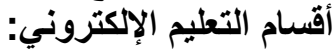

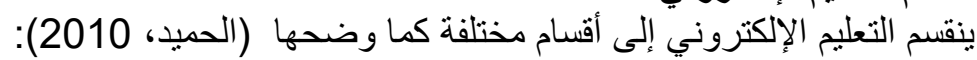

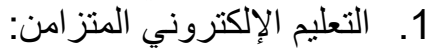

و هو التعليم الإلكتروني الذي يحتاج المتعلمين و المدرس في نفس الوقت حتى تتو افر عملية التفاعل المباشر بينهم.

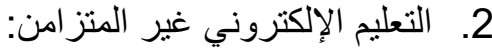

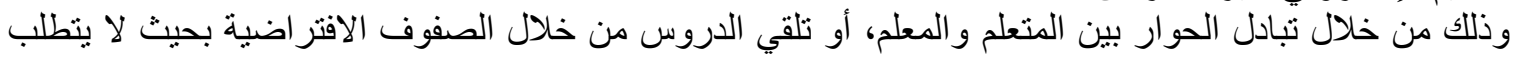

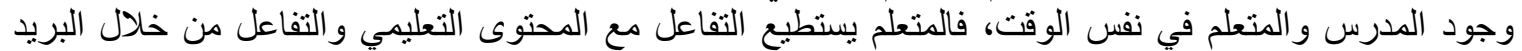

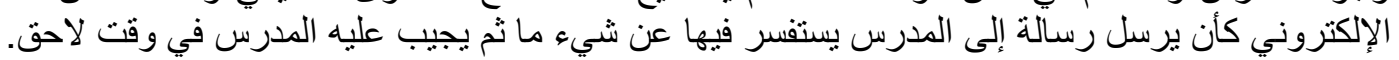

و هو التعليم المدمجي: الذي يستخدم فيه وسائل التئل اتصال معاً لتعلم مادة معينة وقد تشمل هذه الوسائل مزيجاً من الإلقاء المباتشر

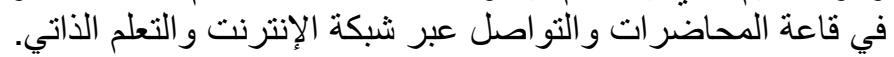

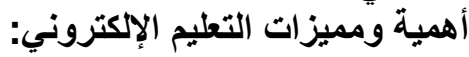
1.يسهم التعليم الإلكتروني في تنمية الإكية التفكير وإثراء عملية التعلم، وتعديل المعلومات و الموضو عات المقدمة فيها وتحديثها

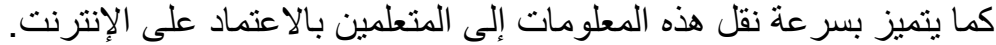

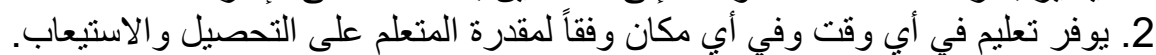

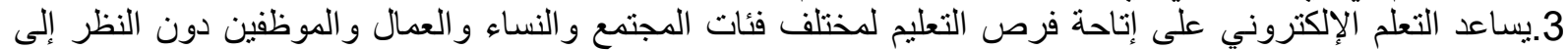

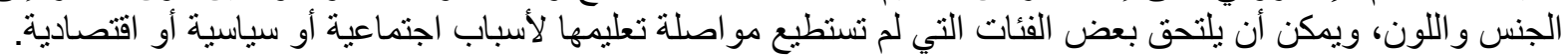

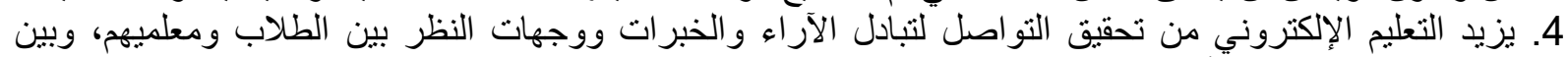

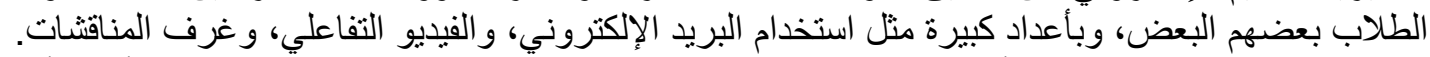

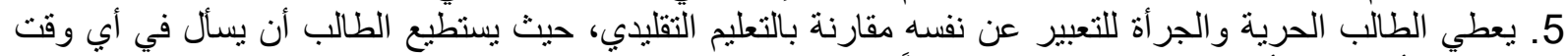

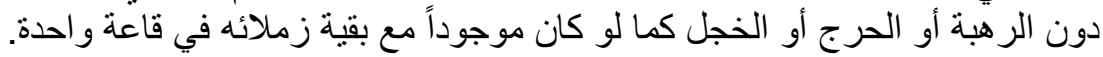

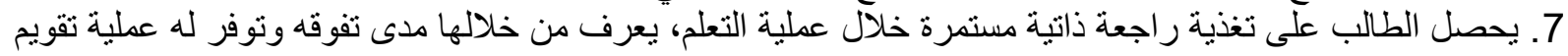

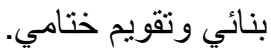

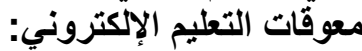
هنالك العديد من المعوقات الأكتروني: التي تحول دون توظيف تكنولوجيا التعليم الإلكتروني في العملية التعليمية وهي كالتالي:

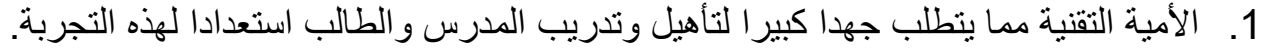

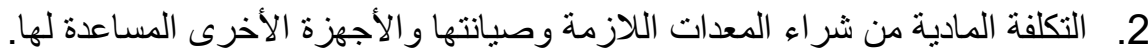

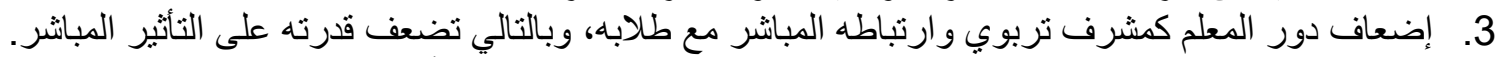

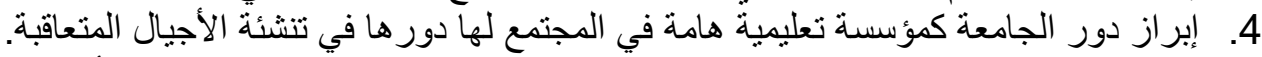

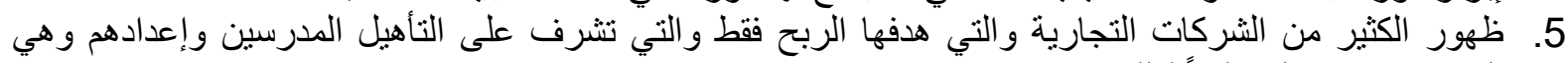
الحقيقة غير مؤهلة علمياً لذلك.

6. كثرة الأجهزة العلمية المستخدمة في العملية التعليمية قد تصيب المتعلمين بالفتور في استخدامها.

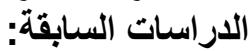
سيتم عرض الدر اسات التي ترتبط بالتعليم بموضوع الدراسة وفق ما تم الاطلاع عليه من در اسات سابقة ذات الصلة على

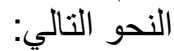

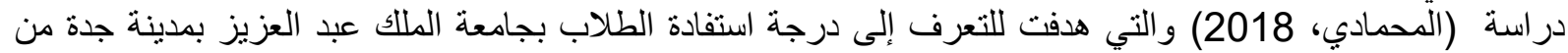

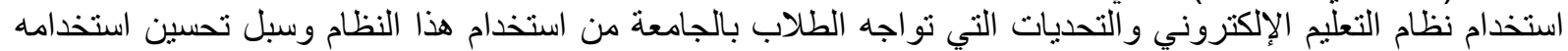

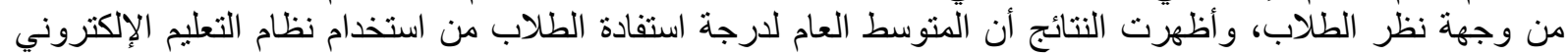

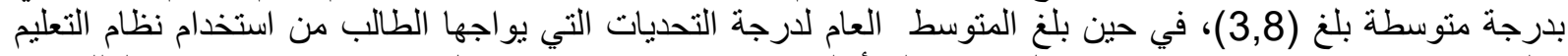

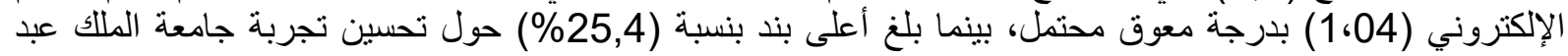

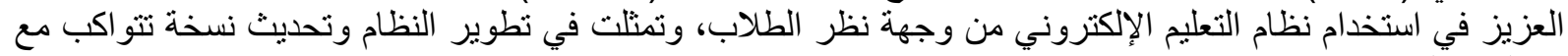




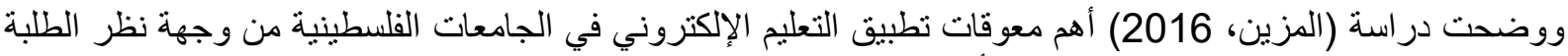

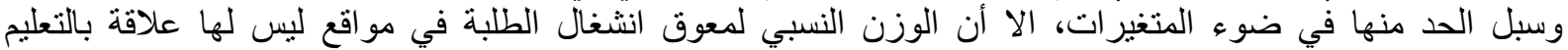

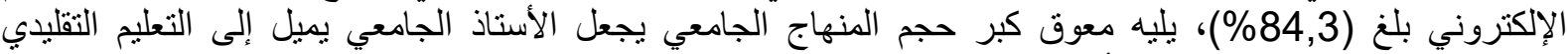

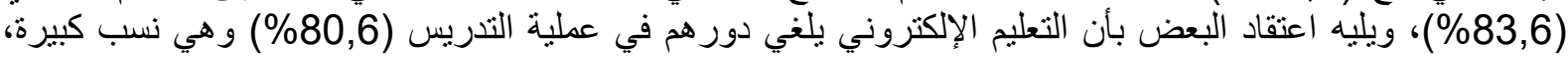

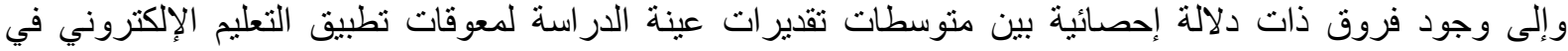

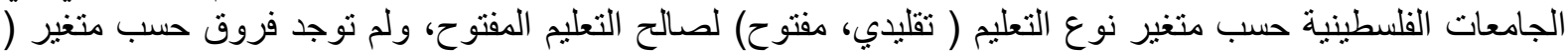

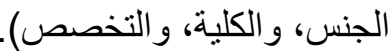

و وأظهرت دراسة (حنتولي، 2016) و اقع التعليم الإلكتروني في جامعة النجاح الوطنية ودوره في تحقيق التفاعل بين

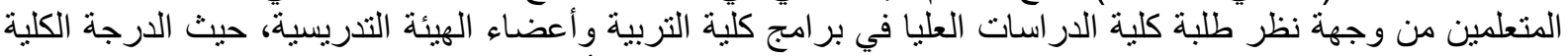

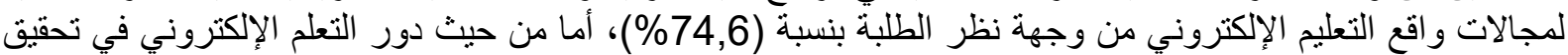

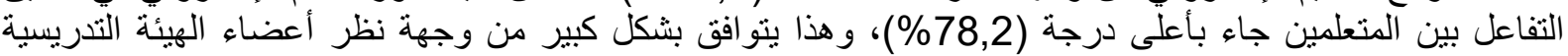

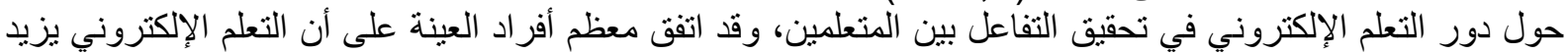

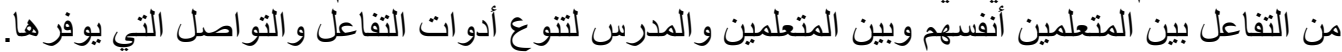

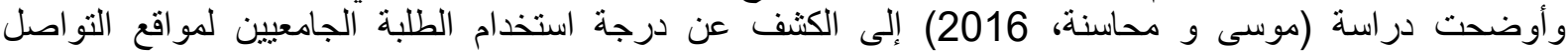

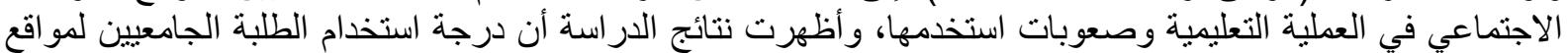

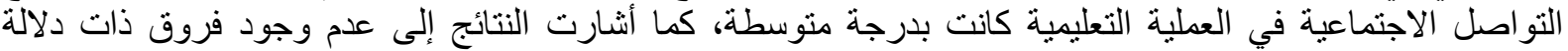

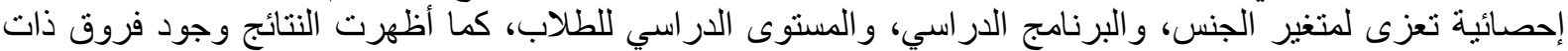

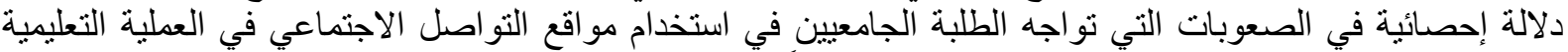

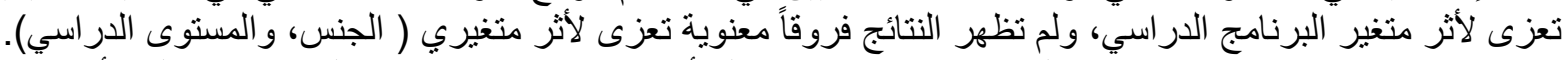

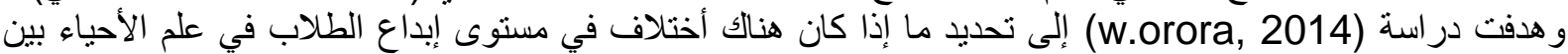

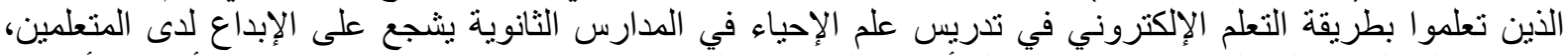

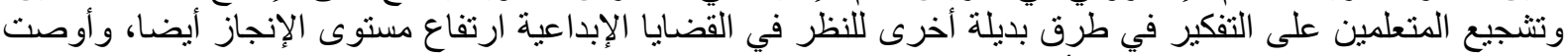

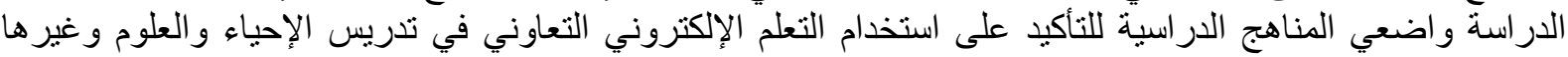
لتحسين فعالية المعلمين.

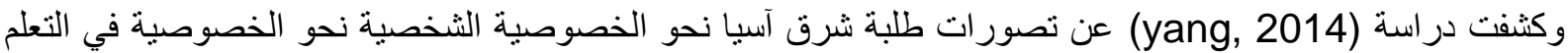

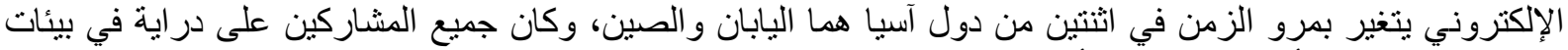

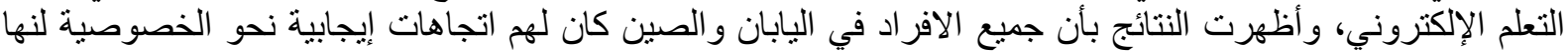

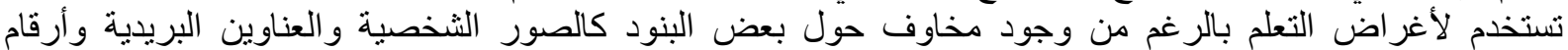

الهو اتف.

تطرقت دراسة (برغوتي، 2002)بعنوان "المدرسة الإلكترونية" (دراسة في المفاهيم و النماذج) إلى نظام التندريس

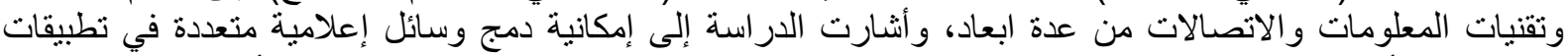

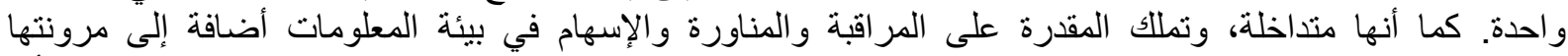

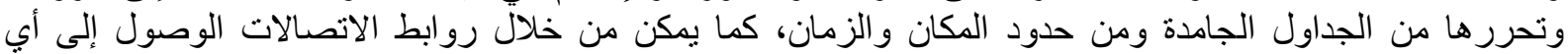

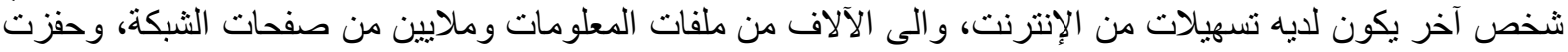

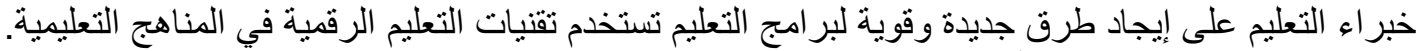

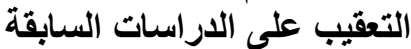
كن خلال استعر اضلي الدراسات السابقة العربية والأجنبية ظهرت الدراسة بمجموعة من أوجه التشابه وأوجه الاختلاف كالتالي: 1.تتشابة الدراسة الحالية مع الدراسات السابقة في المتغير المستقل وهو التعليم الإلكتروني من وجهة نظر الطلبة كما هو عليه.

2. اختلفت الدراسة الحالية عن الدراسات السابقة في بيئة التطبيق وفي في دراسة واقع التعليم على تحقيق التتمية المعرفية

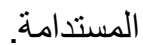

3. تتفق الدراسة الحالية مع الدراسات السابقة من حيث استخدام المنهج الوصفي التحليلي و أداة الدراسة الاستبانة.

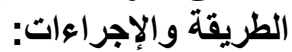

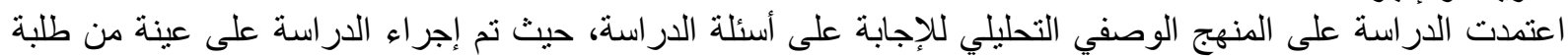
كلية البريمي الجامعية بمحافظة البريمي لمعرفة واقع التعليم الإلكتروني و علاقته بمكونات المعرفة المستدامة. 


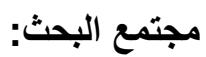

$$
\begin{aligned}
& \text { طلاب كلية البريمي الجامعية البالغ عددهم (3500) طالب } \\
& \text { عينة البحث: } \\
& \text { بلغت عينة الدر اسة وفق معادلة كوشر ان لحساب عينة الدر اسة (347) طالب. } \\
& \text { أداة البحث: }
\end{aligned}
$$

تم تطبيق الاستبانة للحصول على استجابات الطلاب وفق متغيرات الدراسة، ونم إعدادها بعد الرجوع إلى إلى الدراسات

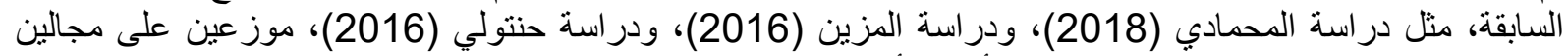

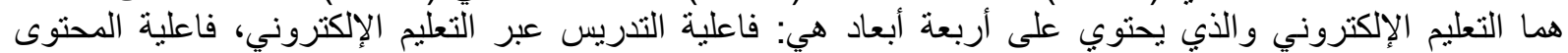

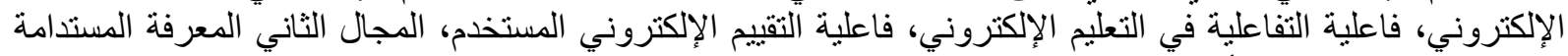
و الذي يحتوي على 4 أبعاد السياسات التربوية، الوسائل والوسائط التكنولوجية، الوقائع والإمكانيات، المخرجات التئية

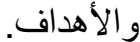

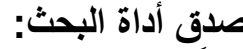

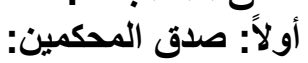

\begin{tabular}{|c|c|c|c|c|}
\hline جداً & قالطا & سططة & (1) & (1) \\
\hline 1 & 2 & 3 & 4 & 5 \\
\hline
\end{tabular}

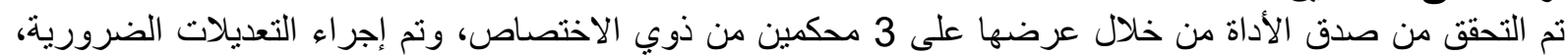

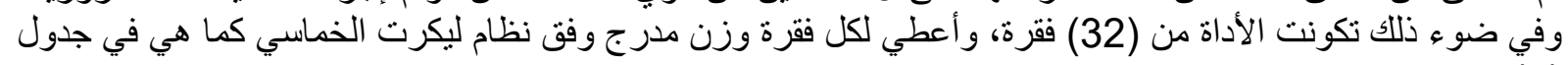

جون 1)

\begin{tabular}{|c|c|c|}
\hline مستوى الدلالة & معامل الارتباط & المجال \\
\hline 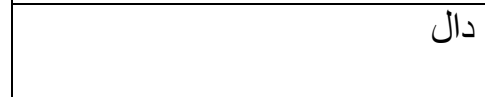 & 0,849 & فالإلكتية التدريس عبر التعليم \\
\hline 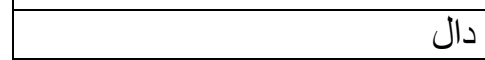 & 0,834 & فاعلية المحتوى الإلكتروني \\
\hline 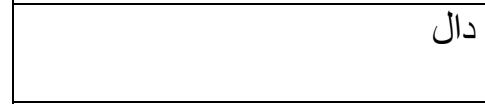 & 0,869 & فالإعترونية التفاعلية $\quad$ في التعليم \\
\hline 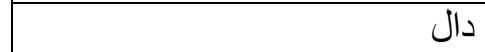 & 0,869 & فاعلية التقييم الإلكتروني المستخدم \\
\hline 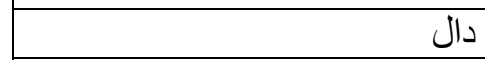 & 0,884 & السياسات التربوية \\
\hline دال & 0,847 & الوسائل و الوسائط التكنولوجية \\
\hline 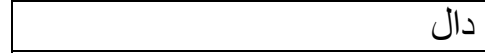 & 0.860 & الوقائع و الإمكانيات \\
\hline دال & 0,801 & المخرجات و الأهداف \\
\hline
\end{tabular}

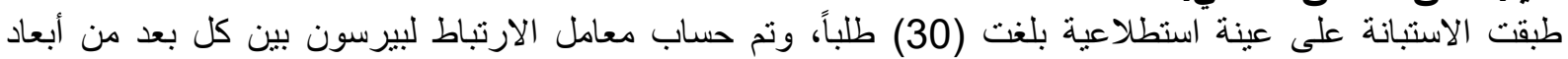

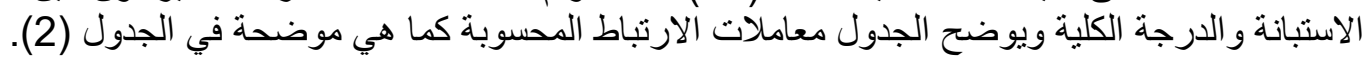

جدول 2 معاملات الارتباط بين كل مجال و الدرجة الكلية لاداة الدر اسة

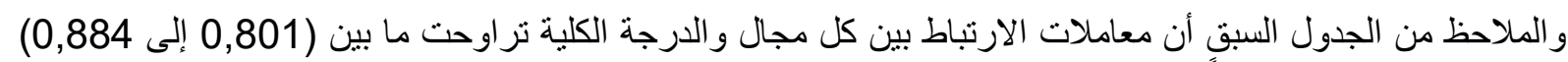
و هي معاملات دالة إحصائياً وقادرة أن على إجراء الاءتات تطبيق الاستبانة على على عينة الدراسة.

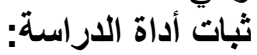

\begin{tabular}{|c|c|c|}
\hline الفا كرونباخ & عدد الفقر ات & 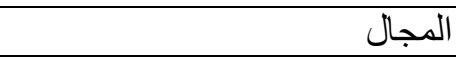 \\
\hline 0,913 & 4 & فاعلية التدريس عبر التعليم \\
\hline & & الإلكتروني \\
\hline 0,860 & 4 & فاعلية المحتوى الإلكتروني \\
\hline 0,835 & 4 & فالإلكترية التفاعلية في التعليم \\
\hline
\end{tabular}
تم التحقق من ثبات الاستبانة باستخدام معامل الثبات ألفا كرونباخ لكل بعد من أبعاد الاستبانة وللأداة ككل ويوضح الجدول

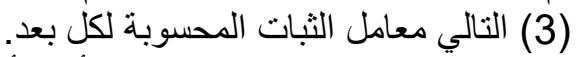
جدول 3 معاملات الثبات المحسوبة لأبعاد أداة الدر اسة والدارجة الدية الكلية 
THE REALITY OF E-LEARNING PERFORMANCE IN THE MINISTRY OF HIGHER

EDUCATION AND ITS RELATIONSHIP TO INDICATORS SUSTAINABLE

KNOWLEDGE (AL BURAIMI UNIVERSITY COLLEGE AS A MODEL)

\begin{tabular}{|c|c|c|}
\hline 0,856 & 4 & فاعلية التقييم الإلكتروني المستخدم \\
\hline 0,858 & 4 & السياسات التربوية \\
\hline 0,817 & 4 & الوسائل و الوسائط التكنولوجية \\
\hline 0,821 & 4 & الوقائع و الإمكانيات \\
\hline 0,930 & 4 & المخرجات و الأهداف \\
\hline 0,861 & 32 & مجمل أبعاد الاستبانة \\
\hline
\end{tabular}

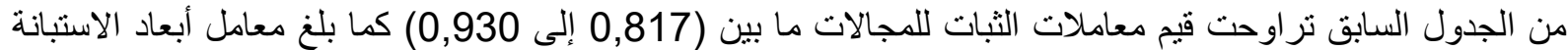
الكلي للأداة (8,61) و هي نسبة ثبات جيدة ويمكن الوثنوق بأداة الاستبانة لجمع البيانات من عينة الدر استة. المعالجات الإحصائية: الإن تم استخدام برنامج الرزم الإحصائية (spss) بعد إدخال البيانات في الحاسب الآلي، وتمت معالجة البيانات باستخدام

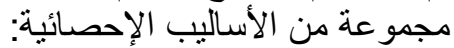
- أختبار الفا كرونباخ لقياس ثبات الأنبات الأداة.

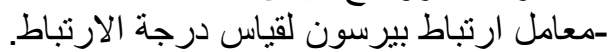
ـ - المنوسطات الحسابية والأوزان النسبية.

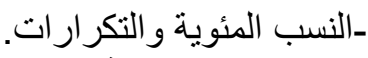
عرض النتائج ومناقشتها: والنيات

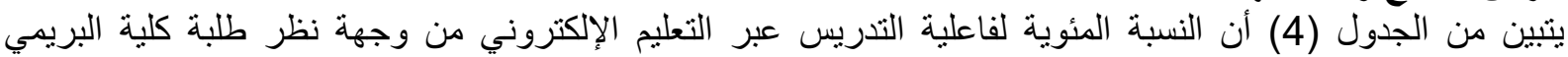

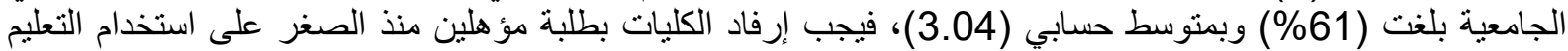

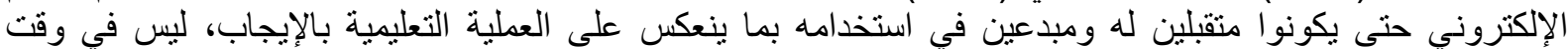

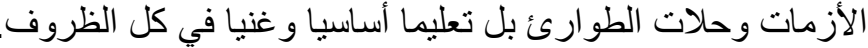

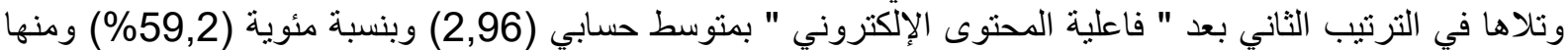

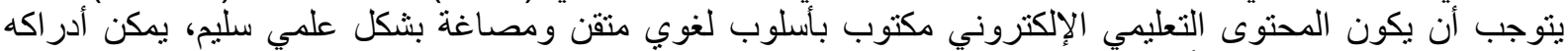

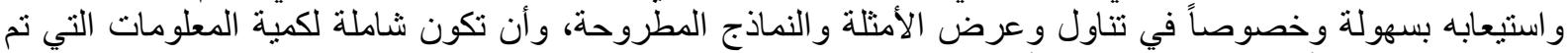

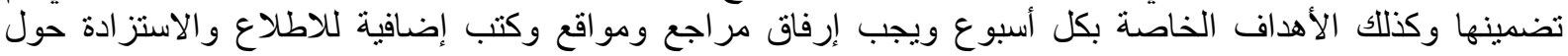

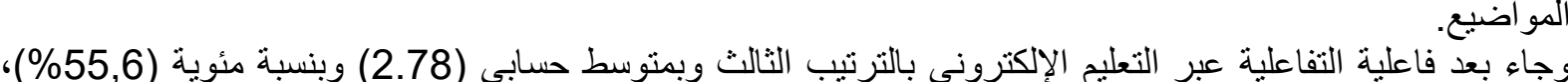

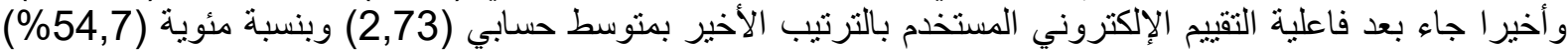
ويعزى ذللك إلى وجوب صياغة المساقات الدراسية وفق نظام تقييمي معين يتناسب مع آلية التعليم الإلكتروني. جدول 4المتوسط الحسابي و الانحر اف المعياري والوزن النسبي لاستجابات عينة الدراسة لأبعاد مجال التعليم الإلكتروني الإني

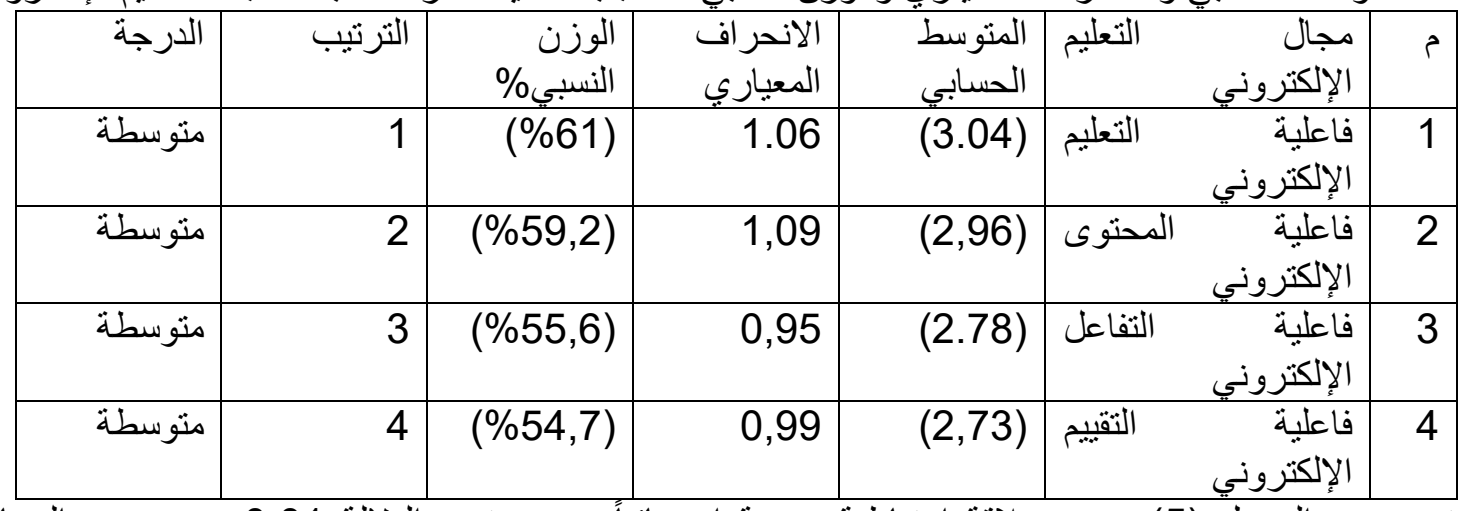

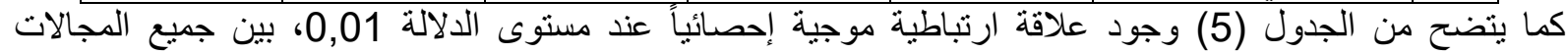

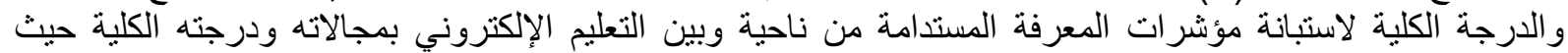

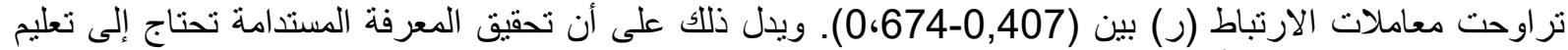

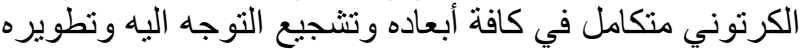


جدول 5العلاقات الارنباطية بين أبعاد الاداة

\begin{tabular}{|c|c|c|c|c|}
\hline \multicolumn{4}{|c|}{ التعليم الإلكتروني } & \multirow[t]{4}{*}{ المعرفة المستدامة } \\
\hline التقييم & فاعلية التفاعلية & التدريس & فاعلية & \\
\hline الإلكتروني & عبر التعليم & التعليم & المحتوى & \\
\hline المستخدم & الإلكتروني & الإلكتروني & الإلكتر & \\
\hline${ }^{* *} 0,674$ & ${ }^{* *} 0,632$ & ${ }^{* *} 0,616$ & ${ }^{* *} 0,646$ & السياسات التربوية \\
\hline${ }^{* *} 0,567$ & ${ }^{* *} 0,546$ & ${ }^{* *} 0,490$ & ${ }^{* *} 0.563$ & التكنولوجئل \\
\hline${ }^{* *} 0,586$ & ${ }^{* *} 0,557$ & ${ }^{* *} 0,529$ & ${ }^{* *} 0,563$ & الو قائع و الإمكانيات \\
\hline${ }^{* *} 0,458$ & $* * 0,479$ & ${ }^{* *} 0,405$ & ${ }^{* *} 0,407$ & المخرجات و الأهداف \\
\hline${ }^{* *} 0,627$ & ${ }^{* *} 0,607$ & ${ }^{* *} 0,559$ & ${ }^{* *} 0,598$ & الدرجة الدات المية المية المتبانة \\
\hline & & & & \\
\hline
\end{tabular}

التوصيات: استتادا إلى ما تم عرضه من نتائج وما تم عرضه في الدراسات السابقة، خلصت الدراسة إلى مجموعة من التوصيات التالية: 1-تأهيل الطلبة و أعضاء هيئة التدريس بكلية البريمي الجامعية من خلال الدورات التدريبية على أيدي مختصين في مجال التعليم الإلكتروني. 2-توفير وزيادة سرعة الإنة الأنترنت لطلبة وأساتذة الكلية مجاناً بالتعاون مع شركات الاتصالات العمانية، لرفع فاعلية المحاضر ات و اللقاءات الإلكترونية.

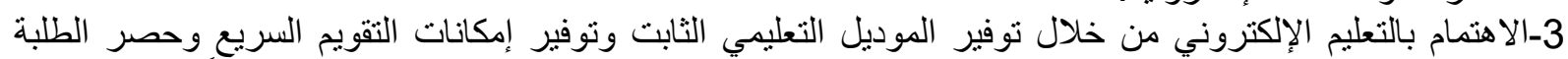

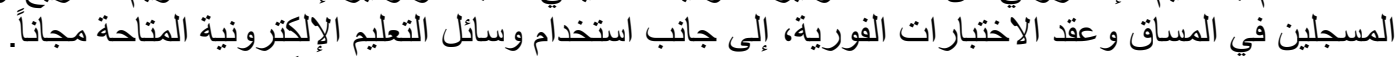

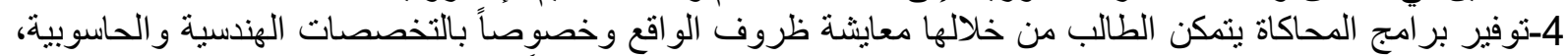
بحيث تمكن الطالب من المحاكاة لتجربة مشرو عنة الذي يعمل عليه قبل تنفيذه فعلياً.

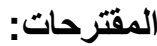

1-در اسة سبل تطوير منظومة التعليم الإلكتروني في كلية البريمي الجامعية. 2-در اسة مقارنة لو اقع التعليم الإلكتروني في كلئية الإكترونيمي في الجامعية مع كليات أو الجية جامعات أخرى.

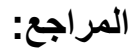

تغريد حنتولي. (2016). و اقع التعلم الإلكتروني في جامعة النجاح الوطنية ودوره في تحقيق التفاعل بين المتعلمين من

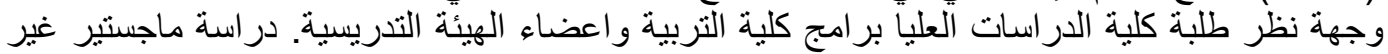

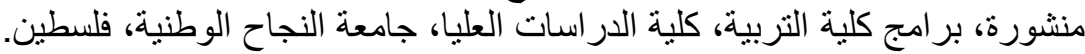
توفيق برغوتي. (2002). المدرسة الإلكترونية. مداخلة القيت في الملتقى الوطني لمركز جيل النيل البحث العلمي حول تقنيات التعليم الحديثة بالمكتة الوظنية.

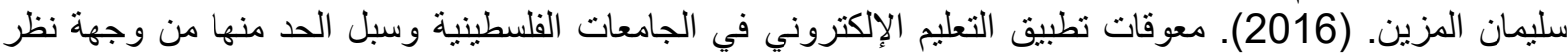

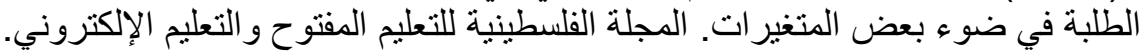

صبري عبد العظيه. (2017). إعداد المعلم في ضو ضو تجارب بعض الدول كلية التربية جامعة حلوان. القاهرة: المجموعة العانية العربية للتندريب و النشر.

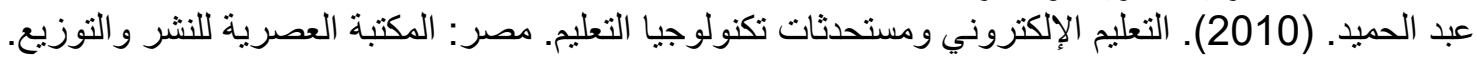

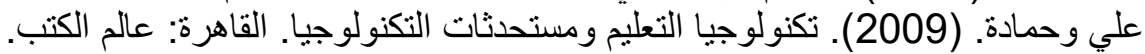

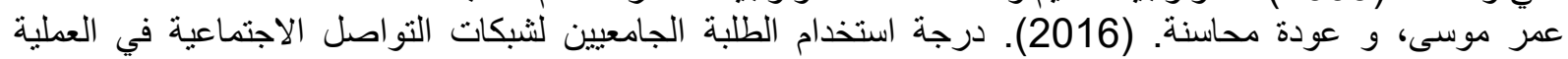

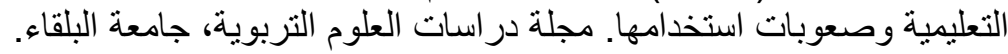

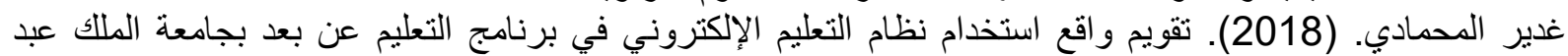

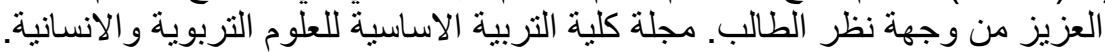

w.orora. (2014). using coopertive e- learning teaching strategy to enhances student creativity in secondary school biologyAstudy of selected school in Nakuru country,kenya. international journal of education and practice.

yang, w. (2014). Student perception toward presonal information and privacy disclosure in e learning. turkish online journal of educational technology. 